\title{
Physical processes in pulsar magnetospheres and non-thermal high-frequency emission
}

\author{
Vladimir V. Usov \\ Department of Physics, Weizmann Institute, Rehovot 76100, Israel
}

\begin{abstract}
The energy source of a pulsar's non-thermal emission is the rotational energy loss of the neutron star. The rotational energy of the neutron star is transformed into the pulsar radiation by a long sequence of processes. The processes of this sequence are discussed.
\end{abstract}

\section{Introduction}

The current sample of radio pulsars contains about six hundred pulsars (e.g., Taylor et al. 1993). The radio luminosities of the pulsars are small compared with the energy loss rate due to the pulsar spin down $\left(\sim 10^{-6}-10^{-5}\right)$. Strong high-frequency radiation, in the optical, $\mathrm{X}$-ray and $\gamma$-ray ranges, is observed from a few radio pulsars (for a review, see Thompson 1994; Hartmann 1995). The observed radiated power for these pulsars is concentrated mainly in the $\gamma$-ray range. The $\gamma$-ray pulsar Geminga is probably also a radio pulsar (Halpern \& Holt 1992) which is "radio quiet" because its radio beam does not intersect the Earth (Ozernoy \& Usov 1977). The pulsar luminosities in $\gamma$-rays are a substantial fraction $\left(\sim 10^{-3}-10^{-1}\right)$ of the spin-down power, $\dot{E}_{\text {rot }}$. This makes studies of high-energy radiation of pulsars a promising avenue to a better understanding of physical processes which result in non-thermal radiation of pulsars.

A common point of all available models of pulsars is that a strong electric field is generated in the magnetosphere of a rotating magnetized neutron star (e.g., Michel 1991). The component of the electric field $E_{\|}=(\mathbf{E} \cdot \mathbf{B}) /|\mathbf{B}|$ along the magnetic field $\mathbf{B}$ is non-zero, and this $E_{\|}$can accelerate particles to ultrarelativistic energies. The accelerated particles emit $\gamma$-rays due to curvature emission and other processes. Some of these $\gamma$-rays are absorbed by creating secondary electron-positron pairs. The created electron-positron pairs screen the electric field $E_{\|}$in the pulsar magnetosphere everywhere except for compact regions. The regions where $E_{\|}$is unscreened are called gaps. These gaps are, in fact, an "engine" which is responsible for the pulsar radiation.

Two kinds of gap models have been popular in trying to understand nonthermal radiation of pulsars. The main difference between these two is the site of gaps. A gap that forms near the magnetic poles of the pulsar is called a polar gap. Besides polar-gap models there are also outer-gap models (e.g., Cheng et al. 1986 a,b; Chiang \& Romani 1994; Usov 1994; Romani and Yadigaroğlu 1995). This review concentrates on the polar gap models mainly (about outer gaps, see Romani, these proceedings). 


\section{The polar gap models}

Polar-gap models for pulsars may be classified in two ways: whether ions or electrons tends to be ejected from the surface, and whether $E_{\|}$is zero or nonzero at the stellar surface. The last depends on the character of particle outflow from the surface.

The sign of the charge of the particles that tend to be ejected from the neutron star surface by the field $E_{\|}$depends on the sign of $\Omega \cdot \mathbf{B}$, where $\Omega$ is the angular velocity of the pulsar rotation. Electrons tend to be ejected for $\boldsymbol{\Omega} \cdot \mathbf{B}>\mathbf{0}$ and ions for $\boldsymbol{\Omega} \cdot \mathbf{B}<0$.

The most familiar model in which there is no ejection of particles from the stellar surface is that of Ruderman \& Sutherland (1975). In this model, the field $E_{\|}$is maximum at the surface and decreases with distance. In contrast, in the model of Arons (1981) it is assumed that charged particles flow freely from the neutron star surface. In this model the electric field $E_{\|}$is equal to zero at the surface, and increases with distance above the surface. A third kind of polar-gap model (Cheng \& Ruderman 1980; Usov \& Melrose 1995, 1996) is an intermediate case where the particles flow from the pulsar surface but not freely. In such a model the field $E_{\|}$is non-zero at the pulsar surface but it is smaller than in the model of Ruderman \& Sutherland (1975).

\subsection{The surface structure and particle ejection}

The structure of matter in the surface layers of neutron stars with $B_{S} \gg \alpha^{2} B_{\mathrm{cr}} \simeq$ $2.35 \times 10^{9} \mathrm{G}$ is largely determined by the magnetic field, and the neutron star surface may be solid, provided that the surface temperature is smaller than the melting temperature (e.g., Liberman \& Johansson 1995; Usov \& Melrose 1995), here $B_{\mathrm{cr}}=m^{2} c^{3} / e \hbar=4.4 \times 10^{13} \mathrm{G}, \alpha=e^{2} / \hbar c=1 / 137$ is the fine structure constant. In this case charges can escape from the surface due to thermionic emission. One may define a characteristic temperature, $T_{\epsilon}($ for $\boldsymbol{\Omega} \cdot \mathbf{B}>0$ ) or $T_{i}$ (for $\boldsymbol{\Omega} \cdot \mathbf{B}<0$ ), such that for a surface temperature $T_{\mathcal{S}}<T_{\epsilon}$ or $T_{\mathcal{S}}<T_{i}$ thermionic emission is unimportant. For a magnetic metal with iron ions and $B \simeq 10^{12}-10^{13} \mathrm{G}$ the characteristic temperature is $T_{\epsilon} \simeq 4 \times 10^{5}\left(B_{S} / 10^{12} \mathrm{G}\right)^{0.4}$ $\mathrm{K}$ for electrons and $T_{i} \simeq 3.5 \times 10^{5}\left(B_{S} / 10^{12} \mathrm{G}\right)^{0.73} \mathrm{~K}$ for ions within a factor of 2 (e.g., Usov \& Melrose 1995).

The flow of charged particles away from the solid surface is very sensitive to the surface temperature for $T_{S} \sim T_{e}$ (or $T_{S} \sim T_{i}$ ). A small change in $T_{S}$ around $T_{s} \sim T_{e}$ (or $T_{s} \sim T_{i}$ ) can have a large effect on the density, $n_{e}$ (or $n_{i}$ ), of outflowing particles, with a change by factor of two causing $n_{e} / n_{\mathrm{GJ}}$ (or $n_{i} Z / n_{\mathrm{GJ}}$ ) to vary from exponentially small (the Ruderman-Sutherland model) to unity (the Arons model), where $n_{\mathrm{GJ}}=|\boldsymbol{\Omega} \cdot \mathbf{B}| / 2 \pi c e$ and $Z$ is the ion charge.

\subsection{Acceleration of outflowing particles}

The Goldreih-Julian density $n_{\mathrm{GJ}}$ is determined so that the electric field $E_{\|}$in the outflowing plasma is screened completely if the charge density is equal to $e n_{\mathrm{GJ}}$. Therefore, the accelerating field $E_{\|}$arises only from deviations from this density. Many causes can lead to such deviations. They are (1) the inertia of particles (Michel 1974), (2) the curvature of the magnetic field lines (Arons 1981), (3) the General Relativity effects (Muslimov \& Tsygan 1992), and (4) the binding of 
particles within the neutron star surface (Ruderman \& Sutherland 1975; Cheng \& Ruderman 1980; Usov \& Melrose 1995, 1996).

When the neutron star surface is cold enough (see 2.1) and there is no ejection of particles from the surface, the binding of particles determines the $E_{\|}$-field distribution in the pulsar vicinity. In the case when particles flow freely from the stellar surface, both the generation of the field $E_{\|}$and acceleration of particles in the polar gaps are mainly because of the General Relativity effects.

\subsection{Generation of $\gamma$-rays}

In a strong magnetic field near the pulsar surface, electrons (and positrons) lose the momentum component transverse to the magnetic field very rapidly and move away from the pulsar practically along the field lines. For such electrons in the ground-state Landau level, any energy loss is negligible up to the Lorentz factors of $\sim 10$. For $10 \lesssim \Gamma \lesssim 10^{2}$, the energy loss due to cyclotron resonant scattering of thermal X-rays from the neutron star surface increases sharply (Dermer 1990). The mean energy of scattered photons is $\sim \hbar \omega_{B} \Gamma \simeq\left(B / 10^{12} G\right)\left(\Gamma / 10^{5}\right)$ $\mathrm{GeV}$, where $\omega_{B}=e B / m c$ and $\Gamma$ is the electron Lorentz factor.

Magnetic Compton scattering is the dominant energy-loss process near the neutron star surface when the electron Lorentz factors are less than $\sim 10^{6}$ for typical $\gamma$-ray pulsar magnetic fields and surface temperatures measured by ROSAT (e.g., Sturner 1995). At $\Gamma>10^{6}$, the main energy loss for ultrarelativistic electrons in the pulsar magnetospheres is due to curvature radiation. In this case the rate of energy loss is $\left|\dot{\varepsilon}_{e}\right|=2 e^{2} c / 3 R_{c}^{2} \Gamma^{4}$, and the mean energy of curvature photons is $\bar{\varepsilon}_{\gamma}=3 \hbar c / 2 R_{\mathrm{c}} \Gamma^{3}$ (e.g., Ochelkov \& Usov 1980), where $R_{\mathrm{c}}$ is the radius of curvature of the magnetic field lines.

For all known pulsars, $\gamma$-rays generated near the neutron star surface are produces in a state below the pair creation threshold (Usov \& Melrose 1995).

\subsection{Propagation of $\gamma$-rays and pair creation}

The conventional expression for the refractive index of a plasma, with the vacuum polarization by the magnetic field taken into account, differs from unity by the order of $\left[0.1 \alpha\left(B / B_{\mathrm{cr}}\right)^{2}+\left(\omega_{p} / \omega\right)^{2}\right] \sin ^{2} \vartheta$, with $\omega_{p}=\left(4 \pi e^{2} n_{p} / m\right)^{1 / 2}$, where $n_{p}$ is the plasma density, $\omega=\varepsilon_{\gamma} / \hbar$ is the photon frequency and $\vartheta$ is the angle between the photon wave vector $\mathrm{k}$ and the magnetic field $\mathbf{B}$ (Erber 1966; Adler 1971). For $\omega \gg 3 \alpha^{-1 / 2}\left(B_{\mathrm{cr}} / B\right) \omega_{p}$, the vacuum polarization gives the main contribution to the difference between the refractive index and unity. This condition is well satisfied for $\gamma$-rays near the pulsar surface. Hence, to understand the process of $\gamma$-ray propagation in the vicinity of pulsars it suffices to consider propagation in the vacuum polarized by a strong magnetic field.

The principal modes of propagation for a photon in the magnetized vacuum are linearly polarized with electric vectors either perpendicular $(\perp$ mode) or parallel ( $\|$ mode) to the plane formed by the photon wave vector $\mathbf{k}$ and the vector $\mathbf{B}$ (e.g., Adler 1971).

While the photon is below the pair creation threshold, $\varepsilon_{\gamma} \sin \vartheta=2 m c^{2}$ for $\|$ mode and $\varepsilon_{\gamma} \sin \vartheta=2 m c^{2}\left\{1+\left[1+\left(2 B / B_{\mathrm{cr}}\right)\right]^{1 / 2}\right\}$ for $\perp$ mode, its main (inelastic) interaction with the magnetic field is a splitting into two photons $\gamma+B \rightarrow \gamma^{\prime}+\gamma^{\prime \prime}+B$ (Adler 1971; Usov \& Shabad 1983; Baring 1991 and references therein). Under the assumption that the dispersion is small, Adler 
(1971) showed that only $\perp$ mode may undergo the decay, $\perp \rightarrow\|+\|$. Towards the threshold, however, the dispersion law differs considerable from the vacuum case, $\omega=|\mathbf{k}| c$, and the small-dispersion assumption is no longer applicable. The decay conditions for the resonant dispersion law were studied by Usov \& Shabad (1983) who showed that the Adler's conclusion remains unaffected by taking the resonant effects into consideration.

The coefficient of photon absorption by decay in the weak-field limit $B \ll$ $B_{\mathrm{cr}}$ is $\sim 0.1\left(B \sin \vartheta / B_{\mathrm{cr}}\right)^{6}\left(\varepsilon_{\gamma} / m c^{2}\right)^{5} \mathrm{~cm}^{-1}$. Recently, it was claimed (Wunner $e t$ al. 1995) that this formula underestimates the correct splitting rate by several orders of magnitude at $B \sim(0.1-1) B_{\text {cr. }}$.

If the strength of the magnetic field at the pulsar poles is high enough, $B_{S} \gtrsim 0.2 B_{\mathrm{cr}}$, most of the $\perp$-polarized photons with $\varepsilon_{\gamma} \lesssim 10^{2} \mathrm{MeV}$ produced near the pulsar surface, are split and transformed into $\|$-polarized photons before the pair creation threshold is reached (Usov \& Shabad 1983; Usov \& Melrose 1995). As a result, the $\gamma$-ray emission recorded from the pulsar vicinity at energies $\varepsilon_{\gamma} \lesssim 10^{2} \mathrm{MeV}$ may be linearly polarized up to $100 \%$. By observing the polarization of the $\gamma$-ray emission of pulsars it would be possible to estimate the strength of the magnetic field near the pulsar surface.

If the photon energy is above the pair creation threshold, the main process by which a photon interacts with the magnetic field is single-photon absorption, accompanied by pair creation: $\gamma+B \rightarrow e^{+}+e^{-}+B$ (Erber 1966; Adler 1971; Baring 1991). In the application to pulsars it is usually assumed that the $\gamma$ quanta produced by the accelerated electrons below the pair creation threshold propagate through the pulsar magnetosphere until they are absorbed by creating free pairs. However, before a photon reaches the threshold for free pair creation it must cross the threshold for bound pair creation. The assumption that the created pairs are free is not valid if the magnetic field is strong enough, specifically for $B>0.1 B_{\mathrm{cr}}$. In such a strong magnetic field, the $\gamma$-quanta emitted tangentially to the curved force lines of the magnetic field are captured near the threshold of bound pair creation and are then channelled along the magnetic field as positronium, that is, as bound pairs (Usov \& Shabad 1985; Shabad \& Usov 1985, 1986; Herold et al. 1985). This positronium may be stable in the polar gaps against both the jonizing action of the electric field and against photo-ionization (Shabad \& Usov 1985; Bhatia et al. 1992; Usov \& Melrose 1995).

The fact that for $B_{S}>0.1 B_{\mathrm{cr}}$ the electron-positron pairs created in the neutron star vicinity are bound may be very important for many physical processes in the pulsar magnetosphere. For example, positronium atoms form a gas of electroneutral particles. Such a gas does not undergo plasma processes, like plasma instabilities, which are responsible for generation of the pulsar radio emission. Maybe, the suppression of free pair creation in strong magnetic fields results in a death line of pulsars at $B_{s} \sim 10^{13} \mathrm{G}$ (Arons 1995, private communication). Besides, unlike free pairs, such bound pairs do not screen the electric field $E_{\|}$near the pulsar. Screening requires a net charge density, which can build up due to free pairs being separated by $E_{\|}$, but cannot build up if the pairs remain bound. As a result the pulsar luminosity is higher than it would be in the absence of formation of positronium (Usov \& Shabad 1985; Usov \& Melrose 1995, 1996 and below). 


\subsection{Non-thermal luminosities}

The total power carried away by both relativistic particles and radiation from the polar gap into the pulsar magnetosphere is

$$
L_{p} \simeq \dot{N}_{\text {prim }} e \Delta \varphi,
$$

where $\dot{N}_{\text {prim }}$ is the flux of primary electrons (or positrons) from the polar cap and $\Delta \varphi$ is the potential across the polar gap. Equation (1) is valid irrespective of whether the electron-positron pairs created near the pulsar are free or bound. The version of pair creation determines only the $\Delta \varphi$ value.

Ruderman \& Sutherland (1975) were the first to develop a self-consistent polar-gap model in which the screening of the field $E_{\|}$by the pairs created in it is taken into account. Consideration of this screening led Ruderman \& Sutherland (1975) to conclude that the potential across the polar gap cannot exceed $\Delta \varphi_{\mathrm{RS}} \simeq$ a few $\times 10^{12} \mathrm{~V}$. This upper limit on $\Delta \varphi$ is valid for any polar-gap model in which free pairs are created by $\gamma$-quanta absorption in the magnetic field.

The density of the primaries cannot be more than $n_{\mathrm{GJ}}$, and therefore, we have the following upper limit on the flux of the primaries, $\dot{N}_{\text {prim }} \leq n_{G J} c \Delta S$, where $\Delta S$ is the surface of the polar cap.

It is convenient to define the ratio $\eta_{\gamma}=L_{p} / \dot{E}_{\text {rot }}$ of the spin-down power going into both high-energy particles and radiation. For $\dot{N}_{\text {prim }}=n_{\mathrm{GJ}} c \Delta S$ and $\Delta \varphi=\Delta \varphi_{\mathrm{RS}}$ the corresponding fraction is (e.g., Usov \& Melrose 1995)

$$
\eta_{\gamma}^{f} \simeq 1.5 \times 10^{-3}\left(\frac{B_{S}}{0.1 B_{\mathrm{cr}}}\right)^{-8 / 7}\left(\frac{P}{0.1 \mathrm{~s}}\right)^{15 / 7}
$$

where $P=2 \pi / \Omega$ is the pulsar period.

In conventional polar-gap models (e.g., Ruderman \& Sutherland 1975; Arons 1981; Cheng \& Ruderman 1980) where created pairs are free the value of $\eta_{\gamma}^{f}$ is more or less the same and differs from (2) by a factor of 2 or so. From Table 1 we can see that $\eta_{\gamma}^{f}$ is more than an order of magnitude smaller than $\eta_{\gamma}^{\text {obs }}=L_{X+\gamma} / \dot{E}_{\text {rot }}$, i.e. the inferred high efficiency of conversion of rotational energy into $\gamma$-ray radiation cannot be explained within the framework of these models.

One suggestion to overcome the contradiction between the polar-gap theory and the $\gamma$-ray observations is that the rotation and magnetic axes of the $\gamma$-ray pulsars are nearly aligned, and the $\gamma$-ray radiation is strongly beamed (Dermer $\&$ Sturner 1994; Daugherty \& Harding 1994). However, these small beam widths imply that the chance of observing any given pulsar from the Earth is too small, about $10^{-2}$ (Daugherty \& Harding 1994)

Recently, Usov \& Melrose $(1995,1996)$ developed the modified polar-gap model that implies a greater power going into primary particles than conventional models, if the production of free pairs is suppressed, as occurs in a sufficiently strong magnetic field, $B>0.1 B_{c r}$. In this model, the fraction of the spin-down power going into both high-energy particles and radiation is

$$
\eta_{\gamma}^{b} \simeq \frac{3}{2}\left(\frac{P}{P_{1}}\right)^{3 / 2}\left[1-\left(\frac{P}{P_{1}}\right)^{3 / 2}\right], \text { where } P_{1} \simeq 0.5\left(\frac{B_{S}}{0.1 B_{\mathrm{cr}}}\right)^{2 / 3} \mathrm{~s} .
$$


Table 1. Properties of $\gamma$-ray pulsars

\begin{tabular}{|c|c|c|c|c|c|c|c|c|}
\hline Name & $\begin{array}{c}P \\
\mathrm{~ms}\end{array}$ & $\begin{array}{c}B_{S} \\
10^{12} \mathrm{G}\end{array}$ & $\begin{array}{c}D \\
\mathrm{kpc}\end{array}$ & $\begin{array}{c}L_{X+\gamma} \\
10^{36} \mathrm{ergs}^{-1}\end{array}$ & $\begin{array}{c}\dot{E}_{\text {rot }} \\
10^{36} \text { erg s}^{-1}\end{array}$ & $\begin{array}{l}\eta_{\gamma}^{\text {obs }} \\
10^{-2}\end{array}$ & $\begin{array}{c}\eta_{\gamma}^{f} \\
10^{-2}\end{array}$ & $\begin{array}{c}\eta_{\gamma}^{b} \\
10^{-2}\end{array}$ \\
\hline PSR $0531+21$ & 33 & 6.6 & 2 & 2.2 & 450 & 0.5 & 0.01 & 1.7 \\
\hline PSR 0540-69 & 50 & 9 & 55 & 0.9 & 150 & 0.6 & 0.02 & 2.3 \\
\hline PSR 0833-45 & 89 & 6.8 & 0.5 & 0.084 & 7 & 1.2 & 0.08 & 4.6 \\
\hline PSR 1706-44 & 102 & 6.3 & 2.8 & 0.3 & 3.4 & 9 & 0.1 & 6 \\
\hline PSR 1509-58 & 150 & 31 & 4.2 & 0.39 & 20 & 2 & 0.04 & 3.6 \\
\hline PSR 1055-52 & 197 & $\begin{array}{c}2 \\
(6)\end{array}$ & 1.8 & 0.024 & 0.03 & 80 & 1 & - \\
\hline Geminga & 237 & $\begin{array}{l}3.3 \\
(6)\end{array}$ & $(0.15)$ & 0.003 & 0.035 & 9 & 1 & $\overline{27}$ \\
\hline
\end{tabular}

At $P=2^{-2 / 3} P_{1} \simeq 0.6 P_{1}, \eta_{\gamma}^{b}$ has a maximum, $\eta_{\gamma}^{b}=3 / 8$. In this case the total luminosity is comparable with the rate of rotational energy loss. The luminosities of all known $\gamma$-ray pulsars can be explained by the modified polar-gap model.

The modified model is valid only if both $B_{s}>0.1 B_{\mathrm{cr}}$ and $P_{2}<P<P_{1}$, where $P_{2} \simeq 0.07\left(T_{s} / 10^{6} \mathrm{~K}\right)^{4 / 11}\left(B_{s} / 0.1 B_{\mathrm{cr}}\right)^{2 / 11} \mathrm{~s}$. For most of the $\gamma$-ray pulsars in Table 1, such a strong magnetic field in the polar gap is suggested by the surface dipolar component inferred from the spin down. For two of the $\gamma$-ray pulsars (PSR 1055-52 and Geminga) the dipolar estimate is slightly below the required value; nevertheless, it is plausible that the field in the polar cap is strong for bound-pair formation in these two cases provided one invokes higher-order multipolar components, or an off-centered dipole.

The Crab-like pulsars (PSR 0531+21 and PSR 0540-69) have periods shorter than $P_{2}$, and the high-frequency radiation from these pulsars cannot be explained in terms of the modified model. For these two Crab-like pulsars, the outer-gap model of Cheng et al. (1986a) seems satisfactory (Ulmer et al. 1994). Moreover, the $\gamma$-ray emission from the Crab pulsar may also be explained in terms of the slot gap model of Arons (1983). However, the slot gaps is an effective source of the energy for non-thermal radiation only for dipole-like magnetic fields.

Some of the particles created near the top edge of the polar gap are stopped by the field $E_{\|}$and then accelerated back to the star. By bombarding the pulsar surface the reversed particles heat it locally in the polar cap region. In the modified polar-gap model the polar-cap temperature is about $T_{e}$ or $T_{i}$, depending on whether electrons or ions escape from the surface. Both $T_{\epsilon}$ and $T_{i}$ are a simple function of either the work function for electrons or the cohesive energy for ions (e.g., Usov \& Melrose 1995). An interesting implication of the modified model for $\gamma$-ray pulsars is that, in principle, information on the binding of particles to the polar cap and of the $B$-field strength in the polar caps may be deduced from $\mathrm{X}$-ray observations. 


\subsection{Non-thermal high-frequency radiation}

The primary particles accelerated in the polar gap move away from the pulsar and generate $\gamma$-rays. Some part of these $\gamma$-rays is absorbed in the pulsar magnetic field creating secondary pairs. The secondaries can repeat this process, which leads to the development of cascades. Gamma-ray emission from such cascades has been studied by Monte Carlo simulations. In these simulations, a pair cascade was initiated by either Compton-scattered photons (Dermer \& Sturner 1994) or curvature photons (Daugherty \& Harding 1994). In both these case the $\gamma$-ray spectra of pulsars were fitted fairly well. If the rotation and magnetic axes are nearly aligned, both broad single-peaked and sharp double-peaked pulse profiles with $\sim 0.4-0.5$ phase separation are formed, in agreement with observations of $\gamma$-ray pulsar pulse profiles.

If the pulsar magnetic field is nearly orthogonal dipole, the polar cascade models have difficulty explaining the interpulse $\gamma$-ray emission of pulsars. This difficulty might be overcome by taking into account that in a polar-gap model not only cascades in the neutron star vicinity may be a source of powerful highfrequency emission. Plasma instabilities may be developed in the outflowing plasma. For example, the cyclotron instability may be developed near the light cylinder of pulsars (Machabeli \& Usov 1979). This instability leads to pitchangles of the plasma particles. As a result, synchrotron radiation has to be generated from the region of development of the cyclotron instability.

\section{Conclusions and discussion}

Many processes, such as generation of electric fields, particle acceleration, generation of $\gamma$-rays and pair creation, which are relevant to the transformation of the rotational energy of the neutron star into high-frequency emission are considered fairly well. While some processes, for example, photon splitting at $B \gtrsim 0.1 B_{\mathrm{cr}}$, have to be studied more carefully.

Using available data on high-frequency radiation of $\gamma$-ray pulsars, some conclusions about validity of the polar-gap models for $\gamma$-ray pulsars may be done by now. For example, the Ruderman \& Sutherland model in which these is no ejection of particles from the stellar surface is ruled out for the $\gamma$-ray pulsars. Indeed, in this model the polar gaps are symmetric and the energy flux into the pulsar magnetosphere is equal to the energy flux to the polar caps. Since practically all energy flux to the polar caps is reradiated as X-ray emission but only a part of the energy carried by relativistic particles into the pulsar magnetosphere may be radiated in the form of $\gamma$-rays, the pulsar $\gamma$-ray luminosity from a polar cap accelerator alone in this model cannot be more than its thermal X-ray luminosity. This is in contradiction with observations.

Most probably, the $\gamma$-ray emission was observed till recently only from peculiar pulsars from which the $\gamma$-ray flux is anomalously high. Such a $\gamma$-ray flux amplification may be because either the rotation and magnetic axes are nearly aligned or the surface magnetic field is very high. Besedes, the $\gamma$-ray emission may be amplified by the outer gap action if the pulsar period is small enough. Maybe, PSR B0656+14 for which the $\gamma$-ray luminosity is small (Ramanamurthly et al. 1996 ) is the first pulsar with a conventional polar gap as a source of the energy for the pulsar $\gamma$-ray emission (cf., Harding et.al. 1993). 


\section{References}

Adler, S.L. 1971, Ann. Phys., 67, 599

Arons, J. 1981, ApJ, 248, 1099

Arons, J. 1983, ApJ, 266, 215

Baring, M.G. 1991, A\&A, 249, 581

Bhatia, V.B., Chopra, N., \& Panchapakesan, N. 1992, ApJ, 388, 131

Cheng, K.S., Ho, C., \& Ruderman, M.A. 1986a\&b, ApJ, 300, 500\&522

Cheng, K.S., \& Ruderman, M.A. 1980, ApJ, 235, 576

Chiang, J., \& Romani, R.W. 1994, ApJ, 436, 754

Daugherty, J.K., \& Harding, A.J. 1994, Ap.J, 429, 32.5

Dermer, C.D. 1990, ApJ, 360, 197

Dermer, C.D., \& Sturner, S.J. 1994, Ap.J, 420, L75

Erber, T. 1966, Rev. Mod. Phys., 38, 626

Halpern, J.P., \& Holt, S.S. 1992, Nature, 357, 222

Harding, A., Ozernoy, L.M., \& Usov, V.V. 1993, MNRAS, 265, 921

Hartmann, D.H. 1995, A\&A Rev., 6, 225

Herold, H., Ruder, H., \& Wunner, G. 1985, Phys. Rev. Lett., 54, 1452

Liberman, M.A., \& Johansson, B. 1995, Physics - Uspekhi, 38, 117

Machabeli, G.Z., \& Usov, V.V. 1979, Soviet Astron. Lett., 5, 238

Michel, F.C. 1974, ApJ, 192, 713

Michel, F.C. 1991., Theory of Neutron Star Magnetospheres, IC:P

Muslimov, A.G., \& Tsygan, A.I. 1992, MNRAS, 255, 61

Ochelkov, Yu.P., \& Usov, V.V. 1980, Ap\&SS, 69, 439

Ozernoy, L.M., \& Usov, V.V. 1977, Soviet Astron., 21, 425

Ramanamurthy, P.V., et al. 1996, ApJ, 458, 755

Romani, R.W. \& Yadigaroğlu, I.-A. 1995, ApJ, 438, 314

Ruderman, M.A., \& Sutherland, P.G. 1975, ApJ, 196, 51

Shabad, A.E., \& Usov, V.V. 1985, Ap\&SS, 117, 309

Shabad, A.E., \& Usov, V.V. 1986, Ap\&SS, 128, 377

Sturner, S.J. 1995, ApJ, 446, 292

Taylor, J.H., Manchester, R.N., \& Lyne, A.G. 1993, Ap.JS, 88, 529

Thompson, D.J. 1994, in Second Compton Symposium, eds. C.E. Fichtel, N. Gehrels, and J.P. Noris, AIP, 304, 57

Ulmer, M.P., et al. 1994, ApJ, 432, 228

Usov, V.V. 1994, ApJ, 427, 394

Usov, V.V., \& Melrose, D.B. 1995, Aust.J.Phys., 48, 571

Usov, V.V., \& Melrose, D.B. 1996, ApJ, in press

Usov, V.V. \& Shabad, A.E. 1983, Soviet Astron. Lett, 9, 212

Usov, V.V. \& Shabad, A.E. 1985, JETP Lett., 42, 19

Wunner, G., Sang, R., \& Berg, D. 1995, ApJ, 455, L51 